\title{
A Pilot Study on the Effect of Pelvic Exercise on Standing Balance in Patients with Incomplete Cervical Spinal Cord Injury
}

\author{
Bo-Ra Choi ${ }^{1}$, Ju-Hyun Kim ${ }^{1}$, Jeong-Uk Lee ${ }^{1}$, Junghwan Kim ${ }^{2 *}$ \\ ${ }^{1}$ Laboratory of Health Science \& Nanophysiotherapy, Department of Physical Therapy, Graduate School, Yongin University, Yongin, \\ South Korea; ${ }^{2}$ Department of Physical Therapy, College of Public Health \& Welfare Yongin University, Yongin, South Korea. \\ Email: junghwankim3@yongin.ac.kr
}

Received December $6^{\text {th }}, 2012$; revised February $4^{\text {th }}, 2013$; accepted March $15^{\text {th }}, 2013$

\begin{abstract}
The purpose of this study was to evaluate the effect of pelvic exercise on standing balance in patients with incomplete cervical spinal cord injury for healthy rehabilitation. 11 patients ( 8 men and 3 women) with levels of incomplete injury between cervical 3 and cervical 6 who were participated in a study measuring the standing balance using an interactive balance system (IBS) before and after pelvic exercise. The index of standing balance after pelvic exercise was significantly lower than before exercise in patients with incomplete cervical cord injury. The greatest improvements were observed in incomplete cervical spinal cord injury with neurologic levels of cervical 3 and 4 with Brown-Sequard syndrome. These results suggest that the pelvic exercise for rehabilitation significantly improved in part standing balance in patients with incomplete cervical spinal cord injury.
\end{abstract}

Keywords: Incomplete Spinal Cord Injury; Standing Balance; Pelvic Exercise; Rehabilitation

\section{Introduction}

Patients with injury of central nervous system such as brain and spinal cord commonly suffer from significant impairments including paralysis, loss of voluntary motor control, and muscle atrophy [1-3]. Improvements in emergency medical management of spinal cord injury (SCI) patients has also led to a higher proportion of patients presenting with incomplete rather than complete neurologic injuries when compared with past statistics [4]. Approximately half of all traumatic SCI cases are cervical lesions [5]. Trauma to the spinal cord is a particularly devastating injury that often results in loss of sensation and voluntary activity below the level of the injury [6]. Incomplete SCI frequently disrupts the afferent and efferent neural pathways involved in co-requisite voluntary and involuntary muscle activation, which are needed for functional standing and walking [7]. A significant number of persons with incomplete SCI can be retrained in the ability to walk [8]. Balance is an important problem for a majority of ambulatory patients with incomplete SCI. Poor balance can contribute to an increase in falls and related injuries $[9,10]$. The importance of the hip muscle has recently been recognized for its role in improvement of static postural stability [11].

"Corresponding author.
From a neuromuscular aspect, all muscle groups of the lumbar-pelvis-hip complex are active in improving static postural stability and assisting in a return to equilibrium after perturbation [12]. However, central neuropathies such as SCI and stroke usually reduce the strength of core muscles and adversely affect the corresponding functional ability for standing [13]. A reduction in core strength often results a reduction in hip extension [14]. In addition, gait deviations commonly observed after SCI include inadequate hip extension in stance because of tightness or spasticity of iliopsoas muscle [15]. Therefore, the reduction in hip extension causes an increased anterior pelvic tilt and poor balance [14]. Physical therapy for SCI primarily concerns restoration of standing balance for walking. Therefore, pelvic exercise training improves the functional abilities of patients and promotes their independent living in their communities. To our knowledge, few studies have been conducted to determine the effectiveness of pelvic exercise in the treatment of balance problems in patients with incomplete cervical SCI. We felt that a need existed for a study investigating the effect of pelvic exercise on standing balance in these patients. We have measured the effect of pelvic exercise on the changes in balance during standing in patients with incomplete cervical SCI, before and after pelvic exercise, using an interactive balance system. 


\section{Materials and Methods}

From November 2010 to December 2011, all patients with standing balance were measured using an interactive balance system before and after pelvic exercise. Measurements were taken from $11($ men $=8$, women $=3)$ incomplete cervical SCI patients. Criteria for inclusion in the study were as follows: 1 ) a less than 1 year of duration of SCI; 2) ability to stand independently for at least 32 seconds without any assistive device; and 3) ability to stand independently with eyes closed for 32 seconds. Exclusion criteria were: 1) Pain during neck active motion; 2) dizziness during eye closing in a standing posture; and 3) presence of orthopedic or neurological conditions in addition to the SCI. All patients were rated D according to the ASIA impairment scale [16]. One session incorporated pelvic exercise (30 minutes). The treatments were performed according to the concept of massage, stretching, and strengthening exercises performed in a regular sequence. The massage and stretching were applied to the muscles of the pelvic anterior tilt (hip flexor, trunk extensor, quadrates lumborum) for improvement of pelvic range of motion [15,17]. Muscle strengthening exercise was applied to the muscles of the pelvic posterior tilt (hip extensor, abdominalis muscle) to reduce pelvic anterior tilt and to increase hip extension. Every participant was evaluated at each session. Each session included the assessment of static standing balance using the interactive balance system (IBS) (Tetrax Inc., Israel). This system uses four separate platforms, each measuring the vertical pressure fluctuations induced by the two heels and toe parts. A pattern analysis of the postural performance of the above measurements was conducted at the following eight positions: head straight with eyes open (NO); head straight with eyes closed (NC); standing on elastic pads with eyes open (PO); standing on elastic pads with eyes closed (PC); head right with eyes closed (HR); head left with eyes closed (HL); head up with eyes closed (HB); and head down with eyes closed (HF). The measuring period in each case was 32 seconds. Fourier transformations were derived from four independent wave signals and presented in the form of a spectrum; these frequency bands were divided into four categories: low $(0.01-0.1 \mathrm{~Hz})$; medium-low $(0.1-0.5 \mathrm{~Hz})$; medium-high $(0.5-1.0 \mathrm{~Hz})$; and high $(1.0 \mathrm{~Hz}$ and above). The F1 (Low frequency; $0.01-0.1 \mathrm{~Hz}$ ) are linked visual control, and they typically dominate normal steady and undisturbed posture. The F2-4 (medium-low; $0.1-0.5 \mathrm{~Hz}$ ) are sensitive to vestibular stress and disturbances. The F5-6 (medium-high; $0.5-1.0 \mathrm{~Hz}$ ) reflect somatosensory activity by the lower extremities and spine. The F7-8 (high; $1.0 \mathrm{~Hz}$ and above) are often induced by dysfunctions in the central nervous system. The weight distribution index (WDI) was measured for weight distribution over the four platforms. The stability index (SI) represents the status of the general postural stability [18]. Standing balance was evaluated twice, once at pre-intervention and once at post-intervention. All patients provided their informed consent to participation in the study, and the protocol for the study was approved by the Committee of Ethics in Research of the University of Yongin, in agreement with the terms of Resolution 5-1-20, December 2006. The existence of a statistical difference between the standing balance before and after pelvic exercise treatment was assessed by performing Wilcoxon's signed-rank test. Statistical significance was set at the 5\% level. SPSS for Windows (version 12.0) was used for analysis in this study.

\section{Results}

We enrolled 11 incomplete cervical SCI patients in our study. Table 1 presents details about the participants. The ages ranged between 22 and 55 years (mean $37.5 \pm$ 11.0 ), three of the patients were female, and all subjects were inpatients in primary rehabilitation (times after injury between 4 and 8 months, mean $6.3 \pm 1.7$ ). Levels of the SCI were between cervical 3 and cervical 6 . Six patients were diagnosed with central cord syndrome and five patients with Brown-Sequard syndrome. Four healthy subjects without neurological and musculoskeletal disorders were also involved as controls for comparison with patients on standing balance. Mean age of the healthy subjects was 31.0 years (SE 28.3; range 29 - 35). A statistical difference was found between the healthy control subjects and all 11 incomplete cervical SCI patients on standing balance (Figure 1). Pelvic exercise led to a substantial improvement in standing balance of the 11 patients. The results were categorized as follows: 1) the medians of all results from the test positions (NO$\mathrm{HF}$ ); and 2) the medians of all results from the each test position (NO-HF) in the frequency ranges F1-F8, WDI, and SI. All standing balance index measurements were not significantly different for all patients, at the level of cervical $3 / 4$ and the level of cervical $5 / 6$ before the treatment. However, all standing balance indexes were significantly higher in Brown-Sequard syndrome subjects than in central cord syndrome patients before the treatment (Figure 2). The medians of all results from the test positions (NO-HF) were as follows: in patients, standing balance index after pelvic exercise was significantly lower than before exercise. The results by classification injury level showed that total subjects had significant decreases of the F1, F2-4, F5-6, F7-8, WDI, and SI. Patients with a level of cervical $3 / 4$ also showed significant decreases in the F1, F2-4, F5-6, F7-8, WDI, and SI. Patients with a level of cervical 5/6 also showed significant decreases in the F1, F2-4, and WDI. The decrease was 
Table 1. General characteristics of patients with incomplete cervical spinal cord injury.

\begin{tabular}{|c|c|}
\hline Variable & SCI patients \\
\hline Age (years) & $37.5 \pm 11.0$ \\
\hline Height (cm) & $172.1 \pm 6.7$ \\
\hline Weight (kg) & $67.5 \pm 7.4$ \\
\hline BMI $\left(\mathrm{kg} / \mathrm{m}^{2}\right)$ & $22.8 \pm 1.2$ \\
\hline \multicolumn{2}{|l|}{ Marital status } \\
\hline Single (number/\%) & $4 / 36$ \\
\hline Married (number/\%) & $5 / 46$ \\
\hline Separated \& divorced (number/\%) & $2 / 18$ \\
\hline \multicolumn{2}{|l|}{ Education completed } \\
\hline$\leq$ High school (number/\%) & $3 / 27$ \\
\hline Some college (number/\%) & $1 / 9$ \\
\hline$\geq$ Bachelor’s degree (number/\%) & $7 / 64$ \\
\hline \multicolumn{2}{|l|}{ Past history } \\
\hline Hypertension or DM & - \\
\hline Times since SCI (months) & $6.3 \pm 1.7$ \\
\hline \multicolumn{2}{|l|}{ Mechanism of injury } \\
\hline Fall (number/\%) & $5 / 45$ \\
\hline Assault (number/\%) & $6 / 55$ \\
\hline \multicolumn{2}{|l|}{ Injury level } \\
\hline Cervical 3/4 (number/\%) & $9 / 82$ \\
\hline Cervical 5/6 (number/\%) & $2 / 18$ \\
\hline \multicolumn{2}{|l|}{ SCI clinical syndrome } \\
\hline Central cord syndrome (number/\%) & $6 / 55$ \\
\hline Brown-Sequard syndrome (number/\%) & $5 / 45$ \\
\hline \multicolumn{2}{|l|}{ Surgery lesion } \\
\hline Anterior (number/\%) & $9 / 82$ \\
\hline Posterior (number/\%) & $2 / 18$ \\
\hline
\end{tabular}

$\overline{\text { Mean } \pm \text { Standard Error; SCI, spinal cord injury; BMI, body mass index; DM, }}$ diabetes mellitus.

greater in subjects with a level of cervical 3/4 than in patients with a level of cervical 5/6 (Figure 3). The results by classification injury syndrome showed that patients with a level of cervical 3/4 with central cord syndrome showed significant decreases in the F1, F2-4, F5-6, and SI. Those in level of cervical 3/4 with Brown-Sequard syndrome also showed significant decreases in the F1, F2-4, F5-6, F7-8, WDI, and SI (Figure 4). The change was greater in subjects with a level of cervical $3 / 4$ with Brown-Sequard syndrome than in subjects with

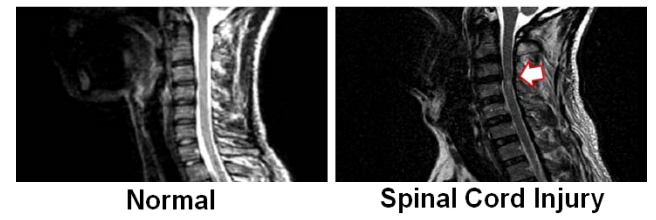

(a)

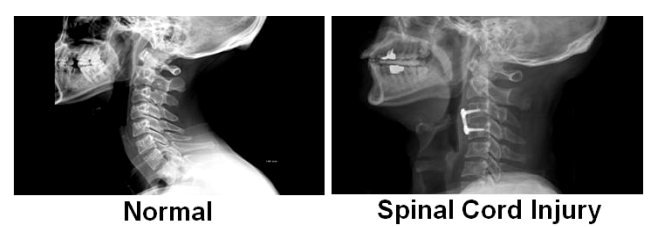

(b)

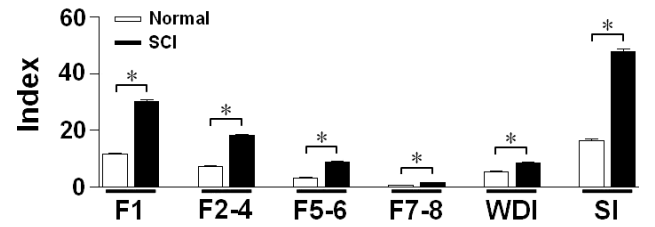

(c)

Figure 1. Differences of MRI (a), X-ray (b), and standing balance index (c) between the healthy subjects and patients with incomplete cervical spinal cord injury. The assessment of standing balance using the interactive balance system (IBS), as described in the Materials and Methods. The F1 are linked visual control system. The F2-4 are sensitive to vestibular stress and disturbances. The F5-6 reflect somatosensory activity by the lower extremities and spine. The F7-8 are linked central nervous system. The weight distribution index (WDI) was measured for weight distribution. The stability index (SI) represents the status of the general postural stability. "Significant differences between normal subjects and SCI with $\mathbf{p}<\mathbf{0 . 0 5}$. SCI, spinal cord injury.

a level of cervical $3 / 4$ with central cord syndrome. The greatest improvements were observed for patients with a level of cervical 3/4 with Brown-Sequard syndrome. The medians of all results from the each test position (NO-HF) were as follows: total subjects with a level of cervical 3/4, $5 / 6$ and subjects with a level of cervical $3 / 4$ with central cord syndrome showed significant decreases in the frequency range $\mathrm{F} 1$ (representing the visual system) in the test position HF (Table 2). Total subjects with a level of cervical $3 / 4$ and subjects with a level of cervical $3 / 4$ with Brown-Sequard syndrome showed significant decreases in the frequency range F2-4 (representing the vestibular system) in test positions $\mathrm{NC}, \mathrm{PC}$, and $\mathrm{HB}$ (Table 2). Most subjects, exclusive of level of cervical 5/6, showed significant decreases in the frequency range F5-6 (representing the somatosensory system) in test positions PC, HR, HB, and HF (Table 3). The most subjects, exclusive of level of cervical 3/4 with central cord syndrome, showed significant decreases in WDI in test position HF. All patients showed significant decreases in SI in test position HB (Table 4). 

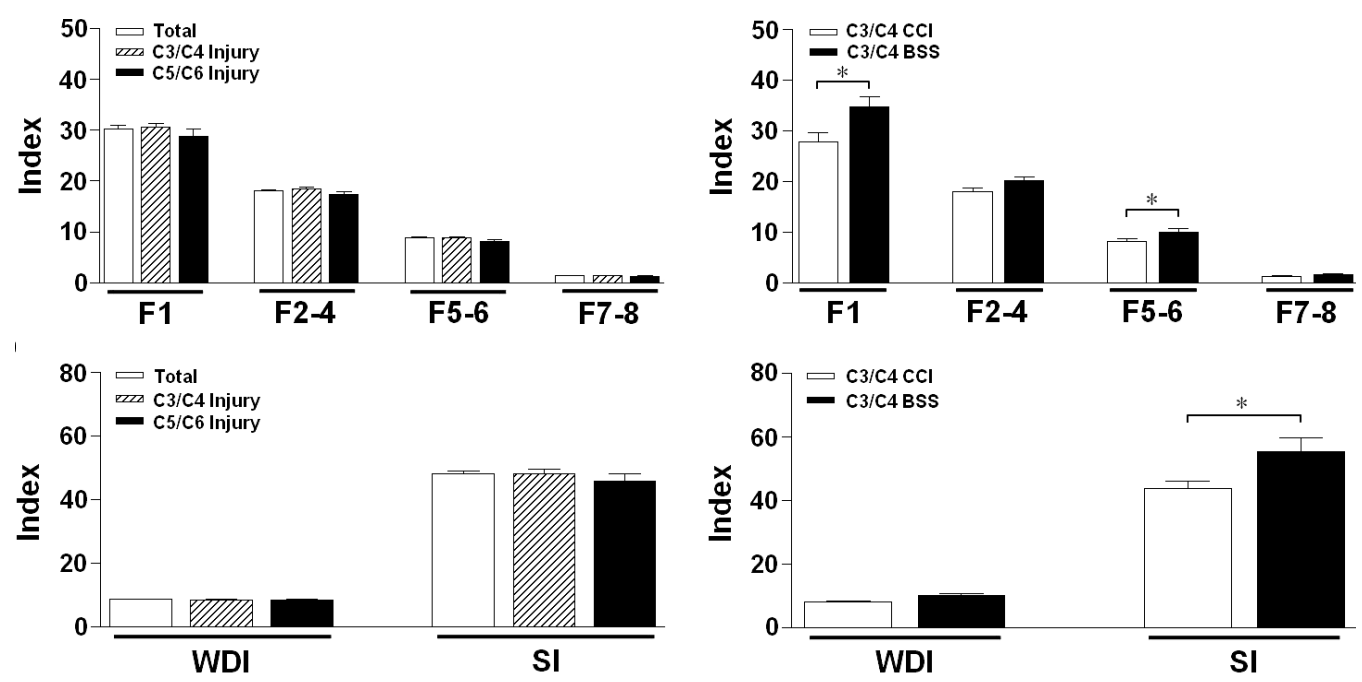

(a)

(b)

Figure 2. Differences of standing balance index before pelvic exercise in patients with incomplete cervical spinal cord injury. ((a) C3/C4 \& C5/C6 injury) Standing balance indexes such as F1, F2-4, F5-6, F7-8, WDI, and SI were measured in both levels of cervical 3/4 and cervical 5/6. ((b) C3/C4-CCS \& BSS) Standing balance indexes were measured in levels of cervical 3/4 in patients with central cord syndrome (CCS) and Brown-Sequard syndrome (BSS). * Significant differences between normal subjects and SCI with $\mathbf{p}<\mathbf{0 . 0 5}$.
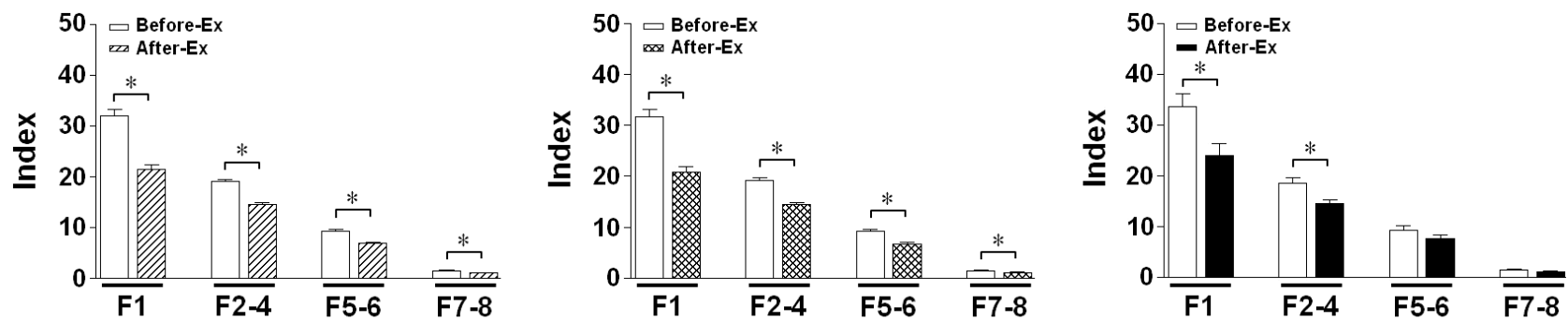

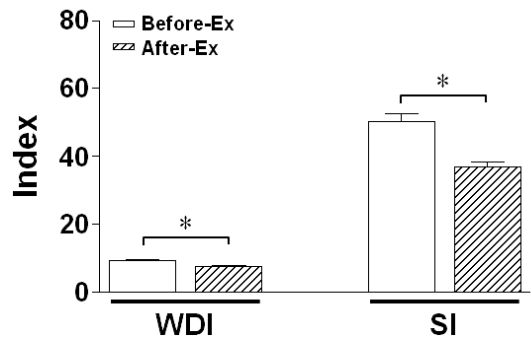

(a)

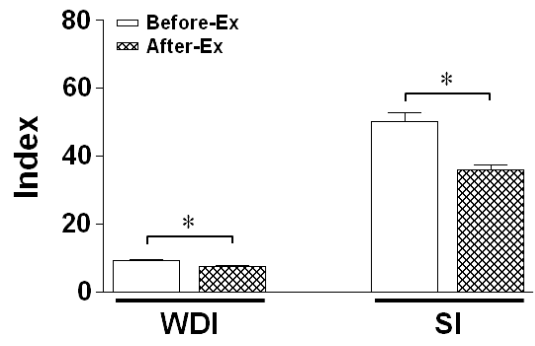

(b)

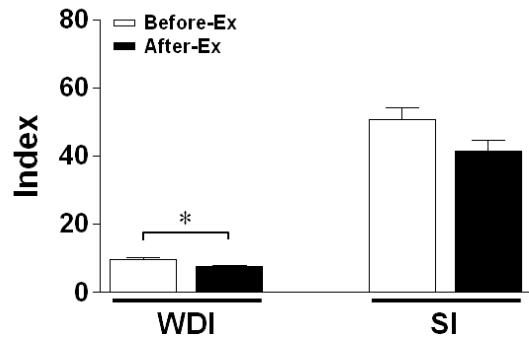

(c)

Figure 3. Effects of pelvic exercise in patients with incomplete cervical spinal cord injury. (a) Standing balance indexes such as F1, F2-4, F5-6, F7-8, WDI, and SI were analyzed in sum total of cervical 3/4 and cervical 5/6 levels before and after pelvic exercise (Ex-Total). Standing balance indexes were analyzed in levels of cervical 3/4 (b) (Ex-C3/C4) and 5/6 (c) (Ex-C5/C6) with or without pelvic exercise, respectively. ${ }^{*}$ Significant differences between before and after pelvic exercise with $p<0.05$. Ex, pelvic exercise.

\section{Discussion}

This study examined the relationship between pelvic exercise and standing balance in patients with incomplete cervical SCI. A limitation for this study is its small sample size, which limits the generalizability of the findings. Therefore, the level of injury (between cervical 3 and 6) and the clinical syndromes (central cord injury, BrownSequard) were controlled. In spite of the small sample, analysis of data produced significant results and provided some new findings regarding the relationship between levels of injury and standing balance. The analysis of the data also revealed a relationship between clinical syndrome and standing balance. Data analysis also divided 

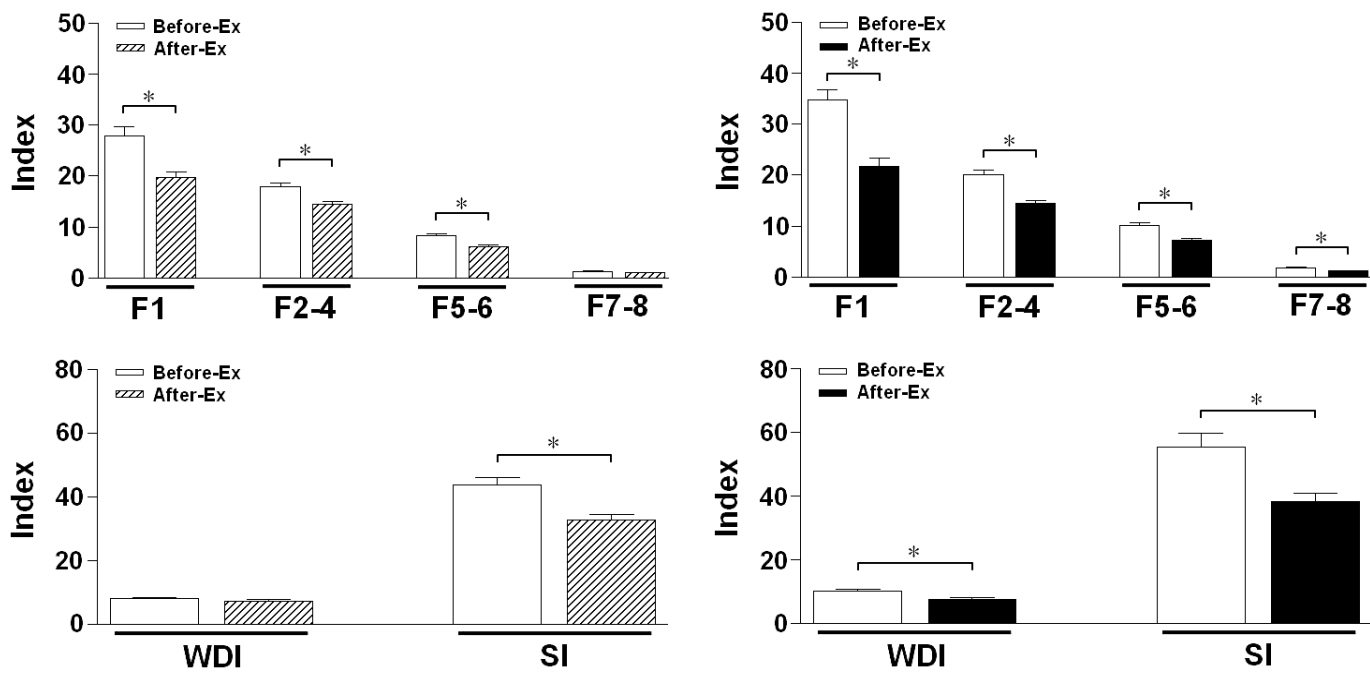

(a)

(b)

Figure 4. Effects of pelvic exercise in levels of cervical 3/4 in patients with central cord syndrome and Brown-Sequard syndrome. Standing balance indexes were analyzed in levels of cervical 3/4 with central cord syndrome ((a) Ex-C3/C4-CCS) and Brown-Sequard syndrome ((b) Ex-C3/C4-BSS), respectively. "Significant differences between before and after pelvic exercise with $\mathrm{p}<0.05$. Ex, pelvic exercise.

each test position (NO-HF) in the frequency ranges F1F8, WDI, and SI. This study was based on hypothesis that pelvic exercise would result in the improved standing balance in patients with incomplete cervical SCI. We showed that pelvic exercise promoted an improvement in standing balance in these patients. All standing balance indexes were significantly lower after the treatment than before. The greatest before session and after session differences were measured in patients with levels of cervical 3/4 and Brown-Sequard syndrome. Incomplete cervical SCI patients exhibit forward bending of the trunk and anterior tilt of the pelvis due to weakened muscle strength [14], shortened muscle length [15], and loss of sensation in the pelvis. This problem may impair ability to maintain standing balance and walking. According to current information, very little is known about the contribution of the lower limbs and upper portions of the body to the adaptation of the gait pattern in patients with incomplete SCI. The main functions of the pelvis and trunk are to maintain body equilibrium and achieve smooth locomotion [19]. The present study showed that all standing balance indexes were significantly lower after the treatment than before. Kim et al. [20] quantified the relationship between individual lower extremity muscle strength (hip flexors/extensor/abductors, knee flexors/extensors, ankle doesiflexors/plantarflexors, and great toe extensors) and functional walking (gait speed, 6-min walk distance, and ambulatory capacity). Their results suggest that walking performance is mainly focused on proximal lower extremity muscle strength, in particular that of hip flexors, hip extensors, and hip ab- ductors. This may imply that proximal lower extremity muscle strength may improve standing balance, walking, and reduction in the fear of falling. In the present study, all participants showed that F2-4 was significantly lower after the treatment than before. Krebs et al. [21] reported that bending the trunk forward during walking has also been reported for subjects with balance deficits due to vestibulopathy. These results suggest that excessive trunk flexion during gait and decreased transverse rotation may increase locomotor stability by positioning the center of gravity more anterior over the base of support. It may imply that reduced pelvic anterior tilt and forward bending of trunk may improve peripheral-vestibular and standing balance. According to Silkwood-Sherer et al. [22], greater pelvic displacement increases somatosensory input though the joints and muscles. In the present study, all participants showed that F5-6 was significantly lower after the treatment than before. For the results of each test position, the frequency range F1 showed significant decreases in the test position HF. The frequency range F2-4 showed significant decreases in the test positions $\mathrm{NC}, \mathrm{PC}$, and $\mathrm{HB}$. The frequency range $\mathrm{F} 5-6$ showed significant decreases in the test positions PC, HR, $\mathrm{HB}$, and HF. The WDI showed significant decreases in the test position HF. The SI showed significant decreases in the test position HB. Looking at these results, decreases in the test positions $\mathrm{NC}, \mathrm{PC}, \mathrm{HR}, \mathrm{HB}$, and $\mathrm{HF}$ were significant. For all of these positions, the eyes were closed. In addition, in the test position PC, the somatosensory input was blocked out. The test positions HR, $\mathrm{HB}$, and HF were associated with vestibular stress. This 


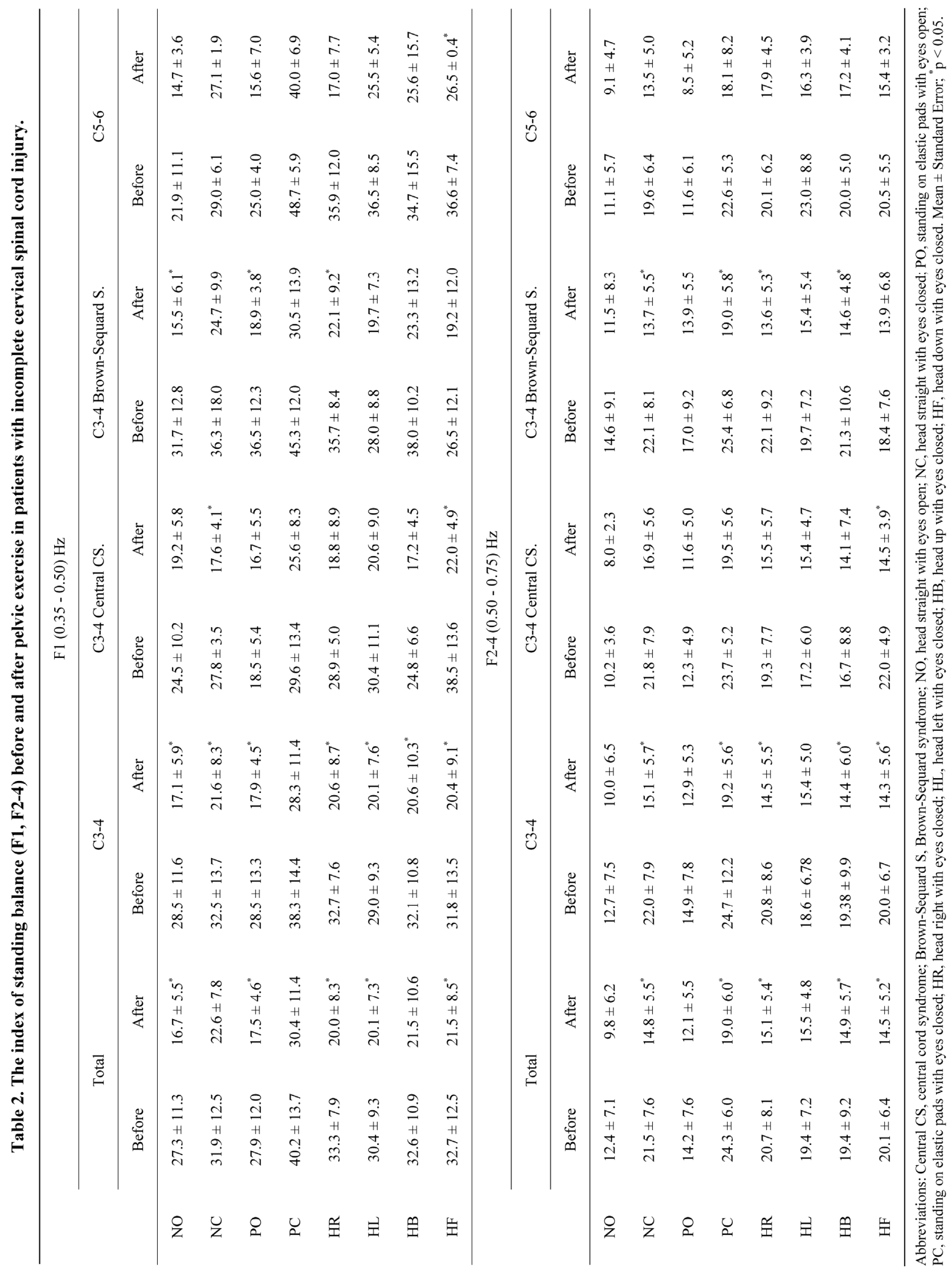




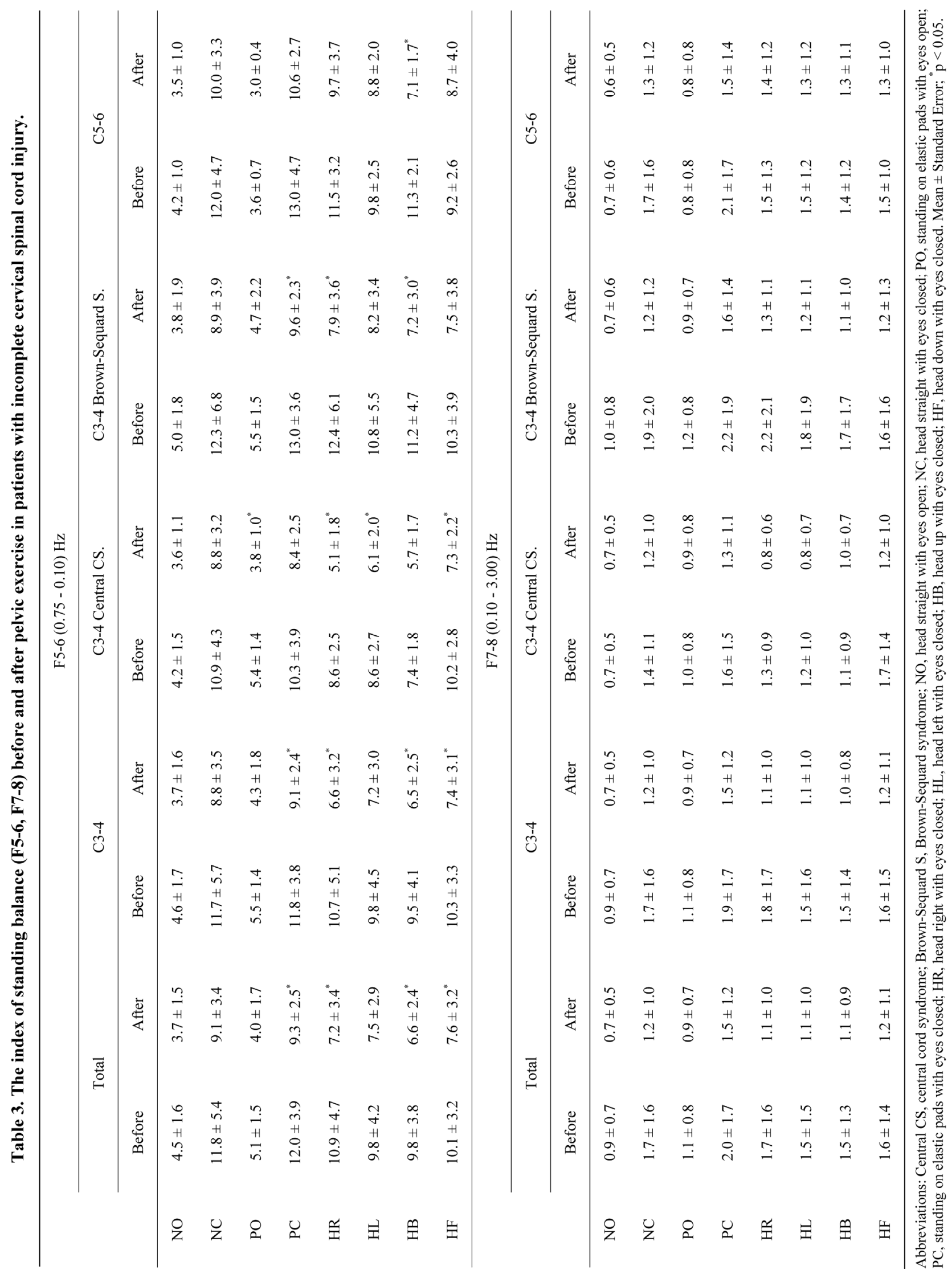




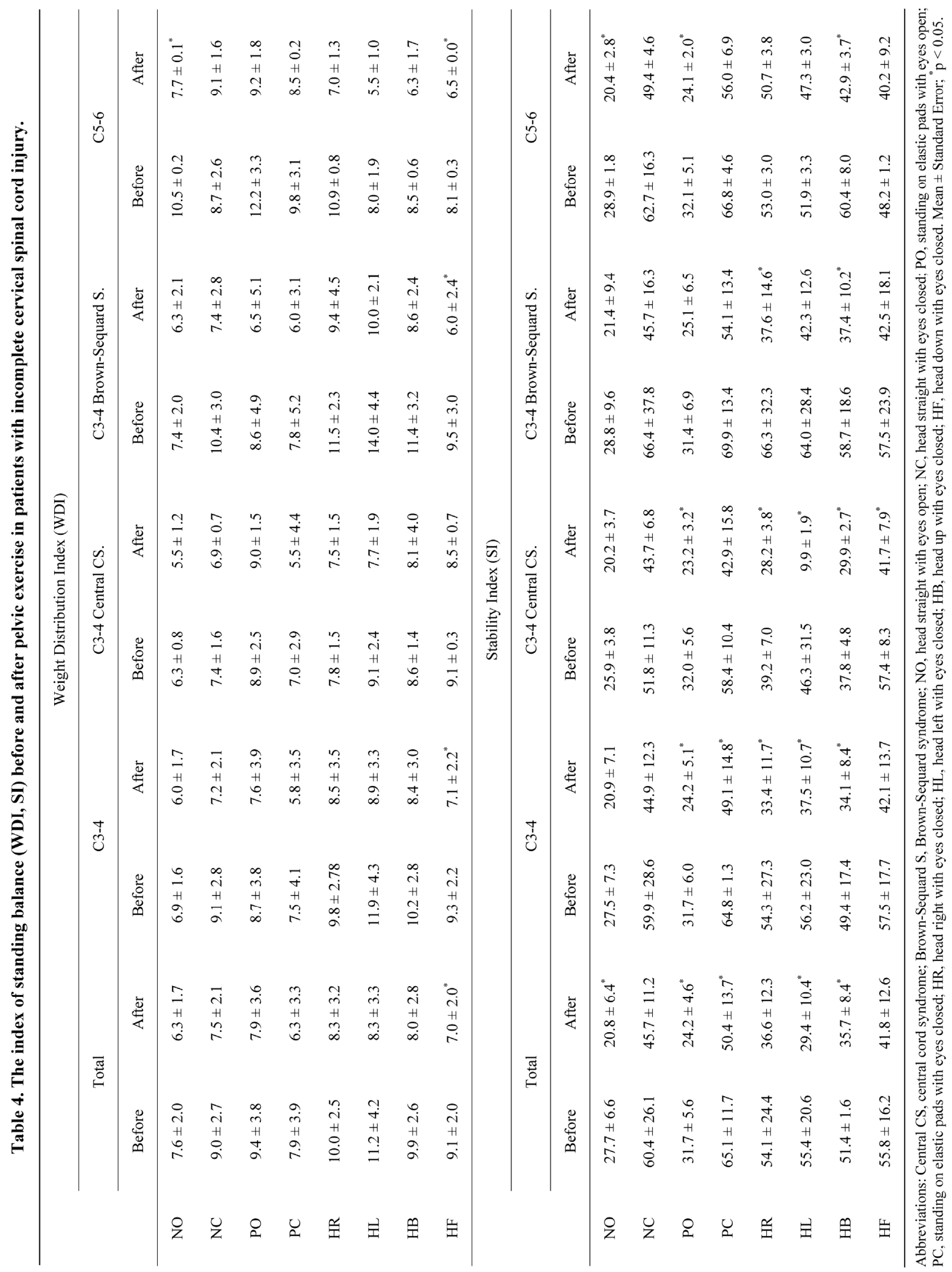


may imply that activation of somatosensory and vestibular functions may compensate for visual deficits. Therefore, this can be explained as activation of the somatosensory and vestibular systems by pelvic exercise. Most previous studies on standing balance have mainly evaluated the effects of therapeutic exercise on somatosensory, visual, and vestibular information. In contrast, the present study evaluated integration of somatosensory, visual, and vestibular effects of pelvic exercise on standing balance. The posture and balance regulation is the result of an integration of various multi-sensory processes. That is, pelvic exercise increased activation of somatosensory, visual, and vestibular information for standing balance. The present study indicated that pelvic exercise training resulted in improved standing balance in patients with incomplete cervical SCI. We showed that pelvic exercise brings about an improvement in the form of a short-term effect on standing balance in incomplete cervical SCI patients. Further studies regarding the duration of the effect are necessary and will be performed with assessment of dynamic standing balance, comparing it with other interventions. In summary, pelvic exercise is a valuable supplement to the conventional physiotherapy approach in standing balance rehabilitation of incomplete cervical SCI patients.

\section{REFERENCES}

[1] M. S. Nash, "Exercise as a health-promoting activity following spinal cord injury," Journal of Neurologic Physical Therapy, Vol. 29, No. 2, 2005, pp. 87-103.

[2] J. H. Kim, M. Y. Kim, J. U. Lee, J. A. Lee, N. M. Yoon, B. Y. Hwang, B. Kim and J. Kim, "The Effects of Symmetrical Self-Performed Facial Muscle Exercises on the Neuromuscular Facilitation of Patients with Facial Palsy," Journal of Physical Therapy Science, Vol. 23, No. 4, 2012, pp. 543-547. doi:10.1589/jpts.23.543

[3] M. Y. Kim, J. H. Kim, J. U. Kim, N. M. Yoon, B. Kim and J. Kim, "The Effects of Functional Electrical Stimulation on Balance of Stroke Patients in the Standing Posture," Journal of Physical Therapy Science, Vol. 24, No. 1, 2012, pp. 77-81. doi:10.1589/jpts.24.77

[4] P. A. Lim and A. M. Tow, "Recovery and Regeneration after Spinal Cord Injury: A Review and Summary of Recent Literature," Annals Academy of Medicine Singapore, Vol. 36, No. 1, 2007, pp. 49-57.

[5] M. J. DeVivo and Y. Chen, "Trends in New Injuries, Prevalent Cases, and Aging with Spinal Cord Injury," Archives of Physical Medicine and Rehabilitation, Vol. 92, No. 3, 2011, pp. 332-338. doi:10.1016/j.apmr.2010.08.031

[6] R. Kizony, L. Raz, N. Katz, H. Weingarden and P. L. Weiss, "Video-Capture Virtual Reality System for Patients with Paraplegic Spinal Cord Injury," Journal of Rehabilitation Research and Development, Vol. 42, No. 5, 2005, pp. 595-608. doi:10.1682/JRRD.2005.01.0023
[7] M. T. Thigpen, J. Cauraugh, G. Creel, K. Day, S. Flynn, S. Fritz, S. Frost, R. Respess, P. Gardner-Smith, M. Brack and A. Behrman, "Adaptation of Postural Responses during Different Standing Perturbation Conditions in Individuals with Incomplete Spinal Cord Injury," Gait \& Posture, Vol. 29, No. 1, 2009, pp. 113-118. doi:10.1016/j.gaitpost.2008.07.009

[8] P. Krawetz and P. Nance, "Gait Analysis of Spinal Cord Injured Subjects: Effects of Injury Level and Spasticity," Archives of Physical Medicine and Rehabilitation, Vol. 77, No. 7, 1996, pp. 635-638. doi:10.1016/S0003-9993(96)90000-3

[9] S. Wydenkeller, M. Liechti, R. Müller and A. Curt, "Impaired Scaling of Responses to Vestibular Stimulation in Incomplete SCI," Experimental Brain Research, Vol. 175, No. 1, 2006, pp. 191-195. doi:10.1007/s00221-006-0688-Z

[10] S. S. Brotherton, J. S. Krause and P. J. Nietert, "A Pilot Study of Factors Associated with Falls in Individuals with Incomplete Spinal Cord Injury," Journal of Spinal Cord Medicine, Vol. 30, No. 3, 2007, pp. 243-250.

[11] F. P. Carpes, F. B. Reinehr and C. B. Mota, "Effects of a Program for Trunk Strength and Stability on Pain, Low Back and Pelvis Kinematics, and Body Balance: A Pilot Study," Journal of Bodywork and Movement Therapies, Vol. 12, No. 1, 2008, pp. 22-30. doi:10.1016/j.jbmt.2007.05.001

[12] J. D. Willson, C. P. Dougherty, M. L. Ireland and I. M. Davis, "Core Stability and Its Relationship to Lower Extremity Function and Injury," Journal of the American Academy of Orthopaedic Surgeons, Vol. 13, No. 5, 2005 , pp. 316-325.

[13] J. S. Petrofsky, E. G. Johnson, A. Hanson, M. Cuneo, R. Dial, R. Somers, G. De La Torre, A. Martinez, M. McKenzie and B. Forrester, "Abdominal and Lower Back Training for People with Disabilities Using a 6 Second abs Machine: Effect on Core Muscle Stability," The Journal of Applied Research, Vol. 5, No. 2, 2005, pp. 345359.

[14] D. C. Kerrigan, L. W. Lee, J. L. Collins, P. O. Riley and L. A. Lipsitz, "Reduced Hip Extension during Walking: Healthy Elderly and Fallers versus Young Adults," Archives of Physical Medicine and Rehabilitation, Vol. 82, No. 1, 2001, pp. 26-30. doi:10.1053/apmr.2001.18584

[15] C. Manella and D. Backus, "Gait Characteristics, Range of Motion, and Spasticity Changes in Response to Massage in a Person with Incomplete Spinal Cord Injury: Case Report," International Journal of Therapeutic Massage \& Bodywork, Vol. 4, No. 1, 2011, pp. 28-39.

[16] F. M. Maynard Jr., M. B. Bracken, G. Creasey, J. F. Ditunno Jr., W. H. Donovan, T. B. Ducker, S. L. Garber, R. J. Marino, S. L. Stover, C. H. Tator, R. L. Waters, J. E. Wilberger and W. Young, "International Standards for Neurological and Functional Classification of Spinal Cord Injury. American Spinal Injury Association," Spinal Cord, Vol. 35, No. 5, 1997, pp. 266-274.

doi:10.1038/sj.sc.3100432

[17] M. A. Diego, T. Field, M. Hernandez-Reif, S. Hart, B. 
Brucker, T. Field and I. Burman, "Spinal Cord Patients Benefit from Massage Therapy," International Journal of Neuroscience, Vol. 112, No. 2, 2002, pp. 133-142. doi: $10.1080 / 00207450212023$

[18] U. Oppenheim, R. Kohen-Raz, D. Alex, A. Kohen-Raz and M. Azarya, "Postural Characteristics of Diabetic Neuropathy," Diabetes Care, Vol. 22, No. 2, 1999, pp. 328-332. doi: $10.2337 /$ diacare.22.2.328

[19] V. P. Stokes, C. Andersson and H. Forssberg, "Rotational and Translational Movement Features of the Pelvis and Thorax during Adult Human Locomotion," Journal of Biomechanics, Vol. 22, No. 1, 1989, pp. 43-50. doi:10.1016/0021-9290(89)90183-8
[20] C. M. Kim, J. J. Eng and M. W. Whittaker, "Level Walking and Ambulatory Capacity in Persons with Incomplete Spinal Cord Injury: Relationship with Muscle Strength," Spinal Cord, Vol. 42, No. 3, 2004, pp. 156-162. doi:10.1038/sj.sc.3101569

[21] D. E. Krebs, D. Wong, D. Jevsevar, P. O. Riley and W. A. Hodge, "Trunk Kinematics during Locomotor Activities," Physical Therapy, Vol. 72, No. 7, 1992, pp. 505-514.

[22] D. Silkwood-Sherer and H. Warmbier, "Effects of Hippotherapy on Postural Stability, in Persons with Multiple Sclerosis: A Pilot Study," Journal of Neurologic Physical Therapy, Vol. 31, No. 2, 2007, pp. 77-84. 